\title{
PRODUTIVIDADE E RENDIMENTO DE SEMENTES DE PINHÃO-MANSO SUBMETIDO À IRRIGAÇÃO E ADUBAÇÃO COM OMM-TECH
}

\author{
ADÃO W. P. EVANGELISTA ${ }^{1}$, PAULO C. DE MELO ${ }^{2}$, EDNALDO L. DE OLIVEIRA ${ }^{3}$, \\ MANOEL A. DE FARIA ${ }^{4}$
}

RESUMO: Com o objetivo de avaliar os efeitos da irrigação e aplicação do fertilizante OrganoMinerais-Marinhos + Biotech $^{\circledR}(\mathrm{OMM}-\mathrm{Tech})$ na produtividade e rendimento do pinhão-manso, um experimento foi instalado em Lavras - MG. O delineamento estatístico utilizado foi o de blocos casualizados, com parcelas subdivididas, contendo três repetições. As parcelas receberam tratamentos constituídos por três formas de aplicação do fertilizante OMM-Tech, ou seja: T1 = Testemunha (Sem OMM-Tech); T2 = via solo (120 $\mathrm{kg} \mathrm{ha}^{-1}$ de OMM-Tech na forma de pó); T3 = via foliar (OMM-Tech na forma líquida e na concentração de 5\%); e T4 = via solo + foliar $\left(60 \mathrm{~kg} \mathrm{ha}^{-1}\right.$ de OMM-Tech na forma de pó + OMM-Tech na forma líquida, na concentração de 2,5\%). As sub-parcelas receberam os tratamentos de manejo de água, ou seja, com e sem irrigação. O sistema de irrigação utilizado foi o gotejamento, com emissores espaçados a cada $0,50 \mathrm{~m}$. Para avaliar a resposta da cultura, utilizaram-se dados de produtividade e rendimento de sementes referentes ao primeiro ano de produção. Os tratamentos aplicados produziram efeitos significativos sobre o rendimento e a produtividade da cultura. As plantas irrigadas e adubadas com OMM-Tech pó, via solo, na dose de $120 \mathrm{~kg} \mathrm{ha}^{-1}$, foram as que apresentaram o melhor resultado.

PALAVRAS-CHAVE: Jatropha curcas L., biodiesel, água.

\section{RESPONSE OF Jatropha Curcas L. CROP TO IRRIGATION AND FERTILIZATION WITH OMM-TECH}

\begin{abstract}
The purpose of this study was to evaluate the effects of irrigation and application of Organo-Minerals-Marine+Biotech (OMM-Tech) in yield and production efficiency of the Jatropha curcas. The experiment was conducted in an experimental area located in Federal University of Lavras, Brazil. A randomized block design with subdivided parcels and three replications was used. The parcels were submitted to different OMM-Tech fertilizer application methods: $\mathrm{T} 1=$ control (no OMM-Tech); T2 = soil application $\left(120 \mathrm{~kg} \mathrm{ha}^{-1}\right.$ of OMM-Tech in a powder form); T3 = leaf application (OMM-Tech in a liquid form with a 5\% concentration); $\mathrm{T} 4=$ soil + leaf application $(60$ $\mathrm{kg} \mathrm{ha}^{-1}$ of OMM-Tech as powder + OMM-Tech in liquid form with a $2.5 \%$ concentration). The subplots received two different water management treatments: with and without irrigation. A drip irrigation system with emitters spaced by $0.50 \mathrm{~m}$ was used. It was evaluated productivity and production efficiency of seeds in the first yield year. The plants irrigated and fertilized with $120 \mathrm{~kg}$ $\mathrm{ha}^{-1}$ of OMM-Tech solid in soil showed better productivity.
\end{abstract}

KEYWORDS: Jatropha curcas L., biodiesel, water.

\footnotetext{
${ }^{1}$ Professor Adjunto, Setor de Engenharia Rural, UFG, Goiânia - GO, awpego@ bol.com.br.

${ }^{2}$ Eng $^{\mathrm{o}}$ Agrônomo, Doutor, Pesquisador, Professor, DAG/UFLA, Lavras - MG, pcmelo@ufla.br.

${ }^{3}$ Professor, IF do Norte de Minas Gerais, Câmpus Araçuaí, ednaldoliberato@ yahoo.com.br.

${ }^{4}$ Prof. Titular, Departamento de Engenharia, UFLA, Lavras - MG, mafaria@ deg.ufla.br.

Recebido pelo Conselho Editorial em: 26-3-2010

Aprovado pelo Conselho Editorial em: 1-3-2011
} 


\section{INTRODUÇÃO}

A busca por sistemas sustentáveis tornou-se meta fundamental para a sociedade como um todo, face às irreversíveis e negativas mudanças ocorridas no ambiente geradas pelo processo de industrialização e seus desdobramentos.

Em face deste quadro, somadas à gradual redução das reservas de petróleo, alternativas produtivas vêm sendo estudadas, visando à obtenção de sistemas economicamente viáveis, socialmente justos, ecologicamente adequados e energeticamente equilibrados. Uma destas alternativas é o biodiesel, sendo que, dentre as culturas energéticas apontadas com grande potencial produtivo de óleo para fins combustíveis, a do pinhão-manso (Jatrofa cuscas L.) apresenta um cenário promissor (FRANCIS et al., 2005).

De acordo com SUJATHA et al. (2008), o pinhão-manso é considerado uma cultura rústica, adaptada às mais diversas condições edafoclimáticas, que sobrevive em condições de solos de baixa fertilidade natural. Contudo, resultados de pesquisa têm revelado que, para se obter alta produtividade de frutos, a planta necessita de água e de solos férteis com boas condições físicas. Logo, a irrigação, a correção da acidez e da fertilidade do solo são pontos decisivos para se obter sucesso e lucratividade com essa cultura.

A busca por novos insumos agrícolas é de grande importância para uma agricultura sustentável e ecologicamente viável. Nesse contexto, é imperativo que se conheçam os fatores que influenciam a disponibilidade de nutrientes para as plantas, advindos da correção do solo e melhoria da sua fertilidade, pelo uso de novos insumos, entre os quais um produto fertilizante, como é o caso do OMM-Tech (MELO \& FURTINI NETO, 2003).

O fertilizante OMM-Tech resulta da mistura do fertilizante organomineral Marinho com o BioTech $^{\circledR}$. O fertilizante Organomineral Marinho é uma alga marinha calcificada, sendo considerado um fertilizante natural, simples, melhorador e condicionador de solos e culturas. Esse fertilizante é obtido sem a adição de qualquer produto químico e, através de processo industrial de secagem e moagem a frio, conserva intactas suas propriedades físicas e químicas (MELO \& FURTINI NETO, 2003). O BioTech ${ }^{\circledR}$ é um bioativador da microbiota do solo, constituído por ácidos orgânicos e complexos enzimáticos, obtidos pela fermentação de tecidos vegetais e biodegradáveis. É considerado fonte de energia para os microrganismos do solo, através da degradação das cadeias orgânicas, sem causar alterações relevantes e irreversíveis na reação do solo e do ambiente rizosférico (ANDRADE et al., 2003).

Além da adubação adequada, o fornecimento de água para a cultura, de forma eficiente, interfere diretamente no processo de produção agrícola. São cada vez mais frequentes os problemas climáticos que atingem severamente as culturas não irrigadas, como a estiagem, mesmo em regiões climaticamente aptas para a maioria das culturas, como o sul do Estado de Minas Gerais, que apresenta períodos de veranico dentro da estação chuvosa. Neste caso, a irrigação é uma técnica que, além de incrementar a produtividade, pode proporcionar a obtenção de um produto diferenciado e de melhor qualidade, com bom preço de mercado. No sul do estado de Minas Gerais, vários pesquisadores constataram um aumento significativo da produtividade em diversas culturas irrigadas, quando comparadas a cultivos não irrigados (AMORIN et al., 2005; CUSTÓDIO et al., 2007; SATO, et al., 2007; SILVA et al., 2008).

Quando plantado no princípio da estação chuvosa, o pinhão-manso inicia a produção de frutos já no primeiro ano de cultivo, atingindo uma produtividade máxima de sementes a partir do quarto ano, com capacidade produtiva potencial por mais de 40 anos. De acordo com informações mais atuais, a planta produz, em média, 100; 500; 2.000 e $4.000 \mathrm{~g}$ de sementes por planta no primeiro, segundo, terceiro e quarto anos de cultivo, respectivamente (TOMINAGA et al., 2007). Dependendo do espaçamento, a produtividade pode passar dos $6.000 \mathrm{~kg} \mathrm{ha}^{-1}$ de sementes. Com essa produtividade, é possível produzir mais de $2.000 \mathrm{~kg} \mathrm{ha}^{-1}$ de óleo. 
Dentro deste contexto, teve-se o objetivo de, neste trabalho, avaliar a produtividade e o rendimento do pinhão-manso em resposta à irrigação e diferentes formas de aplicação de OMMTech.

\section{MATERIAL E MÉTODOS}

O experimento foi desenvolvido no setor de Fruticultura do Departamento de Agricultura da Universidade Federal de Lavras (UFLA). A cidade de Lavras situa-se na região sul do Estado de Minas Gerais, a $21^{\circ} 13^{\prime}$ de latitude sul e $44^{\circ} 58^{\prime}$ ' de longitude oeste, numa altitude média de 918,8 m. A temperatura média anual do ar é de $19,4^{\circ} \mathrm{C}$, e o total anual de precipitação pluvial, de $1.530 \mathrm{~mm}$ (BRASIL, 1992).

As mudas de pinhão-manso provenientes de sementes foram produzidas no setor de Cafeicultura do Departamento de Agricultura da Universidade Federal de Lavras, em Lavras, Minas Gerais. A semeadura foi realizada em tubetes de $120 \mathrm{ml}$, utilizando-se de substrato comercial, seguindo as recomendações de ANEZ et al. (2005). As mudas foram transplantadas em novembro de 2006, no espaçamento de 3,0 x 1,5 m.

O solo da área experimental foi classificado em Argissolo Vermelho eutrófico (EMBRAPA, 1999), de acordo com a Tabela 1.

TABELA 1. Características químicas do solo da área experimental, na camada de 0 a $0,20 \mathrm{~m}$ de profundidade. Soil chemicals characteristics in experimental area, profile of 0 to 0.2 of deep.

\begin{tabular}{|c|c|c|c|c|c|c|c|}
\hline MO & $\mathrm{pH}\left(\mathrm{H}_{2} \mathrm{O}\right)$ & $\mathrm{P}$ & $\mathrm{K}$ & $\mathrm{Ca}^{2+}$ & $\mathrm{Mg}^{2+}$ & $\mathrm{Al}^{3+}$ & CTC \\
\hline $\mathrm{g} \mathrm{kg}^{-1}$ & & \multicolumn{2}{|c|}{------mg dm ${ }^{-3}$} & \multicolumn{4}{|c|}{$-\mathrm{cmol}_{\mathrm{c}} \mathrm{dm}^{-3}$} \\
\hline 2,1 & 6,5 & 6,8 & 101 & 4,0 & 0,9 & 0,0 & 7,1 \\
\hline $\begin{array}{l}\mathrm{V} \\
\%\end{array}$ & $\begin{array}{c}\mathrm{S} \\
\mathrm{mo} \mathrm{dm}^{-3}\end{array}$ & $\begin{array}{c}\mathrm{P}_{\text {remanescente }} \\
\mathrm{Mg} \mathrm{L}^{-1}\end{array}$ & $\mathrm{Zn}$ & Fé & $\mathrm{Mn}$ & $\mathrm{Cu}$ & B \\
\hline 73,1 & 22,7 & 13,6 & 3,6 & 18,2 & 20,3 & 24,6 & 0,4 \\
\hline
\end{tabular}

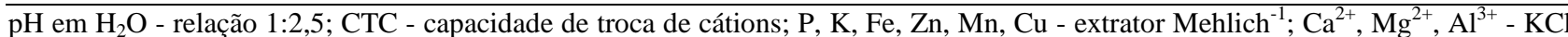
1 mol L ${ }^{-1} ; \mathrm{H}+\mathrm{AL}$ - método $\mathrm{Ca}(\mathrm{OAc})_{2} 0,5 \mathrm{~mol} \mathrm{~L}^{-1}$; pH 7; Matéria orgânica - C orgânico x 1,724 - Walkley Black

O sistema de irrigação utilizado foi por gotejamento, com emissores espaçados a cada $0,50 \mathrm{~m}$. O manejo de irrigação foi realizado monitorando-se a umidade do solo por meio de sensores do tipo watermark, e as lâminas de irrigação foram calculadas de forma a aplicar a quantidade de água necessária para elevar a umidade do solo para a correspondente à capacidade de campo. A uniformidade de irrigação foi avaliada anualmente. Os valores de CUC (coeficiente de uniformidade de Christiansen) mantiveram-se acima de 90\%, nos dois anos analisados.

Foi também realizado um balanço entre o total precipitado e a evaporação do tanque Classe A (ECA), durante a realização do experimento. Para o cálculo da ECA, o coeficiente de tanque (Kp) foi determinado utilizando a equação proposta por ALLEN et al. (1998).

O delineamento estatístico utilizado foi o de blocos casualizados, com parcelas subdivididas, contendo três repetições. As parcelas receberam tratamentos constituídos por três formas de aplicação do fertilizante OMM-Tech, ou seja: T1 = Testemunha (sem OMM-Tech); T2 = via solo (120 $\mathrm{kg} \mathrm{ha}^{-1}$ de OMM-Tech na forma de pó); T3 = via foliar (OMM-Tech na forma líquida, na concentração de 5\%); e T4 = via solo + foliar $\left(60 \mathrm{~kg} \mathrm{ha}^{-1}\right.$ de OMM-Tech na forma de pó + OMMTech na forma líquida, na concentração de 2,5\%). Ressalta-se que esses tratamentos foram definidos com base no experimento sobre nutrição do pinhão-manso com OMM-Tech, desenvolvido por MELO et. al. (2006), no interior de casa de vegetação. As subparcelas receberam os tratamentos de manejo de água, ou seja, com e sem irrigação. As parcelas experimentais foram constituídas por 48 plantas, no qual se consideraram 24 como úteis, perfazendo uma área total de 
$3.456 \mathrm{~m}^{2}$. Os teores de nutrientes presentes no fertilizante OMM-Tech podem ser visualizados na Tabela 2.

TABELA 2. Caracterização química do fertilizante OMM-Tech. Chemical characterization of the OMM-Tech fertilizer.

\begin{tabular}{lc}
\hline Elemento Químico (Macro) & $\mathrm{g} \mathrm{kg}^{-1}$ \\
\hline Cálcio $(\mathrm{CaO})$ & 422 a 455 \\
Magnésio $(\mathrm{MgO})$ & 38 a 53 \\
Silício $\left(\mathrm{SiO}_{2}\right)$ & 21 a 23 \\
Ferro $\left(\mathrm{Fe}_{2} \mathrm{O}_{3}\right)$ & 2,7 a 9,7 \\
Enxofre $(\mathrm{S})$ & 2,5 a 5,2 \\
Fósforo $\left(\mathrm{P}_{2} \mathrm{O}_{5}\right)$ & 0,4 a 1,6 \\
Potássio $\left(\mathrm{K}_{2} \mathrm{O}\right)$ & 0,2 a 0,4 \\
Sódio $(\mathrm{Na})$ & 4,0 a 5,5 \\
Cloro $(\mathrm{Cl})$ & 2,0 a 48 \\
\hline Elemento Químico (Micro) & $\mathrm{mg} \mathrm{kg}^{-1}$ \\
Boro $(\mathrm{B})$ & 8 a 20 \\
Manganês $(\mathrm{Mn})$ & 35 a 200 \\
Molibdênio $(\mathrm{Mo})$ & $<5$ a 5 \\
Zinco $(\mathbf{Z n})$ & $\mathbf{1 1} \mathbf{~ a ~ 2 2 ~}$ \\
Cobalto $(\mathrm{Co})$ & 11 a 16 \\
Vanádio $(\mathrm{V})$ & 14 \\
Níquel $(\mathrm{Ni})$ & 15 \\
Cromo $(\mathrm{Cr})$ & 8 \\
Cobre $(\mathrm{Cu})$ & 21 \\
\hline * Por ser um produto natural, os teores podem apresentar alguma variação.
\end{tabular}

A adubação química no plantio com nitrogênio e fósforo foi realizada na mesma dosagem para todas as parcelas experimentais, com base nos resultados de análise do solo, tomando-se como fundamentação teórica as recomendações de adubação para a cultura da mamoneira, de acordo com LANGE et al. (2005) e SAVY FILHO (1997), por ser uma cultura da mesma família do pinhão-manso, também produtora de sementes ricas em óleo. Tal procedimento foi adotado por não existir até o momento recomendações de adubação específicas para a cultura do pinhão-manso.

As plantas invasoras foram manejadas conforme a necessidade e de acordo com o grau de infestação.

A colheita das parcelas foi feita de forma manual e após a derriça, e homogeneização dos frutos colhidos, as amostras foram acondicionadas em sacaria de fio plástico trançado e submetidas à secagem em bancadas de madeira até atingirem umidade na faixa de 7\%, com base em peso. Em seguida, foram retirados $500 \mathrm{~g}$ de fruto de pinhão-manso para o beneficiamento e, posteriormente, foram determinados: o peso do pinhão-manso beneficiado, a umidade, a produtividade ( $\mathrm{kg}$ por hectare) e o rendimento (litros de fruto por $\mathrm{kg}$ de semente). Para avaliar a resposta da cultura, utilizaram-se dados de produtividade e rendimento de sementes referente ao primeiro ano de produção, ou seja, entre o período de um e dois anos após o transplantio das mudas.

Os resultados foram submetidos à análise de variância, que, quando apresentou resultados significativos, as diferenças entre os tratamentos foram verificadas de acordo com teste de Tukey, ao nível de $5 \%$ de probabilidade.

\section{RESULTADOS E DISCUSSÃO}

Os valores de precipitação (P) e evaporação do tanque Classe A (ECA) para Lavras estão dispostos na Figura 1, durante o período de realização do experimento (11-2007 a 11-2008), 
indicando haver concentração das precipitações para a região de Lavras, entre os meses de novembro a março, quando os valores precipitados superaram consideravelmente a ECA, enquanto nos meses de abril a outubro a precipitação foi inferior à ECA, caracterizando-se déficit hídrico no período; essas informações estão de acordo com DANTAS et al. (2007), ao relatarem que a estação chuvosa para Lavras se estende de outubro a março, e a seca compreende os meses de abril a setembro.

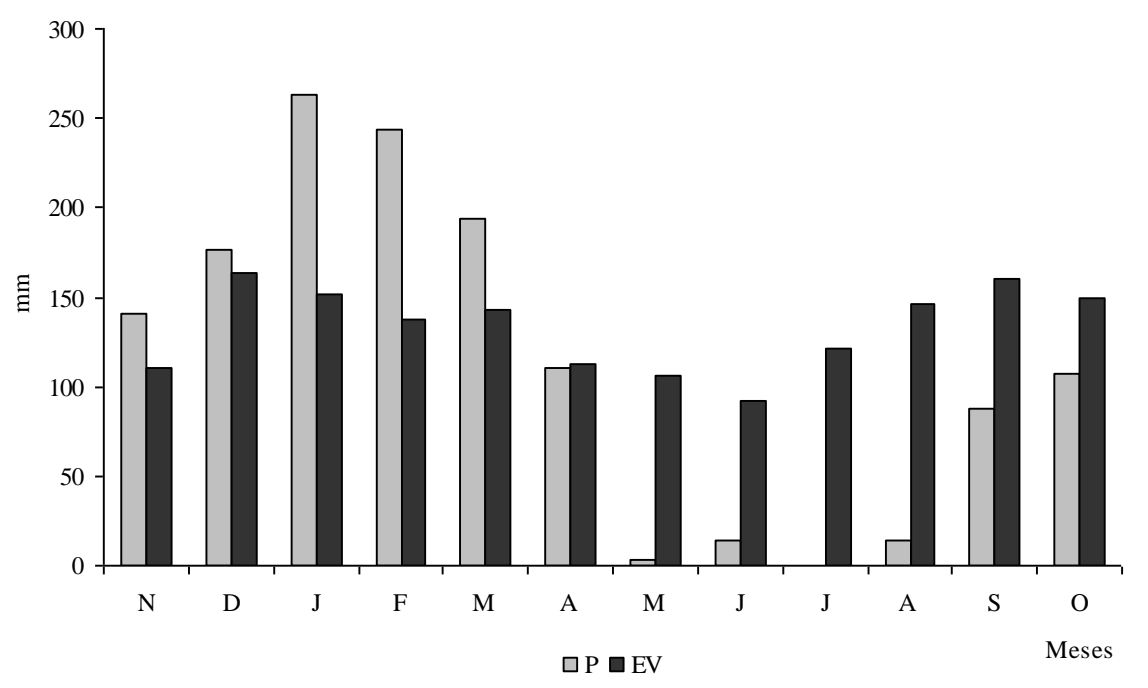

FIGURA 1. Totais mensais (mm) para precipitação (P) e evaporação do tanque Classe A (ECA) ocorridos no período de 11-2007 a 11-2008. Dados fornecidos pela Estação Climatológica Principal do INMET, Lavras - MG. Monthly total rainfall (P) and Class ' $A$ ' Pan evaporation (ECA) in the period of 11/2007 to 11/2008. INMET Climatologic Station - Lavras, MG, Brazil.

O balanço entre a ECA e a precipitação mostra déficit hídrico na ordem de 552,0 mm para os períodos de abril a outubro de 2008. Esse déficit, por outro lado, é função da ausência ou dos baixos valores precipitados entre os meses de junho a setembro e de uma elevada demanda d'água pela atmosfera, o que pode ser constatado através dos valores da ECA, superiores a 5,0 mm dia ${ }^{-1}$ (Figura 1). Ressalta-se que um déficit hídrico superior a $150 \mathrm{~mm}$ pode comprometer a produção da maioria das culturas. Com bases nesses dados, pode-se justificar, então, a irrigação do pinhão-manso na região de Lavras, mesmo essa região apresentando precipitação média anual de $1.500 \mathrm{~mm}$ (BRASIL, 1992).

Verificou-se que houve diferenças significativas entre os tratamentos, em nível de 5\% de probabilidade, para as características de produção analisadas. Entretanto, somente houve efeito significativo da interação entre as diferentes formas de aplicação de OMM-tech e as lâminas de água aplicadas para a produtividade. Essa resposta confirma os dados de LAVIOLA \& DIAS (2008) e ARIZA-MONTOBBIO \& LELE (2010), segundo os quais, existe grande probabilidade de resposta do cafeeiro à adubação e teor de água na região do sistema radicular, o que contradiz a rusticidade da cultura relatada por vários pesquisadores (ARRUDA et al., 2004; SUJATHA et al., 2008; NÚÑEZ-COLÍN \& GOYTIA-JIMÉNEZ, 2009).

Observa-se, na Tabela 3, que o tratamento irrigado foi superior ao não irrigado, com obtenção de produtividade e rendimento de 236,2 $\mathrm{kg} \mathrm{ha}^{-1}$ e 9,01 $\mathrm{L} \mathrm{kg}^{-1}$ contra 83,87 kg ha-1 e 9,97 $\mathrm{L} \mathrm{kg}^{-1}$ das plantas de sequeiro. Ressalta-se que, apesar de a produtividade das plantas irrigadas terem superado as plantas de sequeiro, os valores obtidos foram relativamente baixos, quando comparados aos alcançados por DRUMOND et al. (2010), que observaram na região semiárida do Nordeste brasileiro, que a produtividade do pinhão-manso foi de $2.000 \mathrm{~kg} \mathrm{ha}^{-1}$ de sementes. Ressalta-se que, neste trabalho, a análise foi realizada com dados do primeiro ano de produção, e, portanto, 
provavelmente a planta ainda não tinha atingido seu pleno potencial produtivo. Por outro lado, DRUMOND et. al. (2007) obtiveram produtividades já no primeiro ano de cultivo, em Petrolina$\mathrm{PE}$, variando de $246 \mathrm{~kg} \mathrm{ha}^{-1}$, em condições de sequeiro, a $871 \mathrm{~kg} \mathrm{ha}^{-1}$, em área irrigada. Ressalta-se que a diferença entre os resultados obtidos neste trabalho com aqueles encontrados por DRUMOND et. al. (2007) pode ser explicada pela diferença de localidade, como também podem estar relacionados às variáveis de crescimento da cultura, ou seja, o crescimento inicial da cultura na região sul de Minas Gerais pode ter apresentado taxas menores quando comparadas àquelas da região nordestina brasileira.

TABELA 3. Resultados do teste de comparação de médias, para produtividade $\left(\mathrm{kg} \mathrm{ha}^{-1}\right)$ e rendimento do pinhão-manso $\left(\mathrm{L} \mathrm{kg}^{-1}\right)$ em função dos tratamentos aplicados. Results of averages comparison test, for productivity $\left(\mathrm{kg} \mathrm{ha}^{-1}\right)$ and yield of Jatropha curcas $\left(\mathrm{L} \mathrm{kg}^{-1}\right)$, to all treatments.

\begin{tabular}{lccc}
\hline Tratamentos & & Produtividade $\left(\mathrm{kg} \mathrm{ha}^{-1}\right)$ & Rendimento $\left(\mathrm{L} \mathrm{kg}^{-1}\right)$ \\
\hline Irrigação & $\mathrm{I}$ & $236,2 \mathrm{a}$ & $9,01 \mathrm{a}$ \\
& $\mathrm{S}$ & $83,87 \mathrm{~b}$ & $9,97 \mathrm{~b}$ \\
\hline Formas de aplicação de OMM-tech & $\mathrm{T}$ & $117,92 \mathrm{a}$ & $10,86 \mathrm{a}$ \\
& $\mathrm{F} 1$ & $192,02 \mathrm{~b}$ & $7,88 \mathrm{~b}$ \\
& $\mathrm{~F} 2$ & $183,75 \mathrm{~b}$ & $9,56 \mathrm{c}$ \\
& F3 & $146,43 \mathrm{ab}$ & $9,66 \mathrm{~d}$ \\
\hline
\end{tabular}

"Valores seguidos de letras iguais não diferem estatisticamente; I - irrigado; S - sequeiro; T - testemunha; e F1, F2 e F3 - formas de aplicação de OMM-Tech 1, 2 e 3, respectivamente.

Com relação às formas de aplicação de OMM-tech, verificou-se que as médias de produtividade observadas, quando se utilizaram as três maneiras de aplicação do fertilizante, foram consideradas estatisticamente iguais, porém com grande diferença quando comparadas à média da testemunha (sem adubação). Neste caso, a forma de aplicação de OMM-Tech pó via solo, na dose de $120 \mathrm{~kg} \mathrm{ha}^{-1}$, promoveu a maior produtividade $\left(192,02 \mathrm{~kg} \mathrm{ha}^{-1}\right)$, seguida da forma via foliar $\left(183,75 \mathrm{~kg} \mathrm{ha}^{-1}\right)$ e via solo + foliar $\left(146,43 \mathrm{~kg} \mathrm{ha}^{-1}\right)$. A produtividade das plantas que não receberam adubação foi de $117,92 \mathrm{~kg} \mathrm{ha}^{-1}$. O efeito positivo do OMM-tech sobre a produtividade de sementes de pinhão-manso também foi encontrado por MENDONÇA et al. (2006) e pode ser explicado pela característica do organomineral Marinho como fertilizante e condicionador de solo, que juntamente com o Biotech $^{\circledR}$ (ativador da microbiota do solo) promovem o enriquecimento do solo em nutrientes, por meio da ativação da microbiota e por reações de troca desencadeadas pelos ácidos orgânicos e complexos enzimáticos, que promovem uma disponibilização mais rápida de macro e micronutrientes e elementos raros de fácil liberação.

Verifica-se ainda, na Tabela 3, que o rendimento das plantas submetidas às três formas de aplicação do fertilizante OMM-Tech apresentou resultados considerados estatisticamente diferentes. A testemunha apresentou o pior rendimento, tendo sido necessários 10,9 litros de pinhão-manso na forma de fruto para produzir $1 \mathrm{~kg}$ de sementes. Semelhantemente à produtividade, a forma de aplicação de OMM-Tech via solo na forma de pó e na dosagem de $120 \mathrm{~kg} \mathrm{ha}^{-1}$ foi a que promoveu o melhor rendimento $\left(7,88 \mathrm{~L} \mathrm{~kg}^{-1}\right)$, seguida da forma via foliar $\left(9,56 \mathrm{~L} \mathrm{~kg}^{-1}\right)$ e via solo + foliar $\left(9,66 \mathrm{~L} \mathrm{~kg}^{-1}\right)$. É possível que este fato tenha ocorrido em razão de a forma de aplicação de OMMTech via solo na dosagem de $120 \mathrm{~kg} \mathrm{ha}^{-1}$ ter ocasionado aumento das reservas de fotoassimilados e, por consequência, ter aumentado o desenvolvimento dos grãos no período analisado, o que interferiu positivamente no rendimento. Estes resultados confirmam os relatos de LAVIOLA \& DIAS (2008) que citam que, apesar de o pinhão-manso ser uma cultura rústica, ela responde em produção à adubação, e como a colheita dos frutos de pinhão-manso extrai elevada quantidade de nutrientes do solo, caso estes não forem adequadamente repostos pela adubação, poderá ocorrer empobrecimento do solo ao longo dos anos de cultivo e, por consequência, queda na produção. 
$\mathrm{Na}$ Tabela 4, observa-se que o fator irrigação teve influência significativa sobre a produtividade de sementes de pinhão-manso. Neste caso, as plantas que receberam irrigação e adubação de OMM-tech via solo, na dose de $120 \mathrm{~kg} \mathrm{ha}^{-1}$, foram as que apresentaram, em média, a maior produtividade $\left(313,5 \mathrm{~kg} \mathrm{ha}^{-1}\right)$. Entretanto, quando não se aplicou água, observou-se que os tratamentos de adubação foram considerados estatisticamente iguais. Desta forma, destaca-se a grande importância do fertilizante OMM-Tech e da irrigação no sentido de propiciar melhores condições de produção do pinhão-manso na região de Lavras. Ressalta-se que a região sul de Minas Gerais apresenta períodos de veranico dentro da estação chuvosa, que pode comprometer o desenvolvimento e a produtividade da cultura. Durante o desenvolvimento deste trabalho, foram visualizados sintomas de déficit hídrico nas plantas que não receberam irrigação, como murcha e desfolha. PONCIANO DE DEUS (2010) e OLIVEIRA et al. (2006), em pesquisa realizada com pinhão-manso irrigado no sul de Minas Gerais, também visualizaram os mesmos sintomas de déficit hídrico nas plantas, em épocas de veranico, ocorridos na estação chuvosa. Por outro lado, ARIZAMONTOBBIO \& LELE (2010) relatam que o florescimento do pinhão-manso pode ser induzido duas ou três vezes ao ano, por meio do manejo da irrigação, induzindo déficit hídrico durante um período suficiente para provocar a queda de metade das folhas da planta, para, em seguida, restabelecer a irrigação gradualmente; logo, como o florescimento é um dos principais estágios fenológicos para a produção de óleo de Jatropha curcas, uma vez que o número de flores femininas e sua fecundação determinam quantos frutos e sementes serão desenvolvidos, ressalta-se novamente a importância do uso de irrigação, visando à obtenção de sucesso com essa cultura.

TABELA 4. Interação das lâminas de irrigação e formas de aplicação de OMM-Tech para a produtividade de sementes. Interaction between irrigation levels and fertilization levels with OMM-Tech to seed yield.

\begin{tabular}{lcccc}
\hline \multirow{2}{*}{ Manejo de Água } & \multicolumn{4}{c}{ Formas de Aplicação de OMM-Tech } \\
\cline { 2 - 5 } & Testemunha & Via solo & Via foliar & Via solo + foliar \\
\hline Irrigado & $142,68 \mathrm{cA}$ & $313,5 \mathrm{abA}$ & $276,86 \mathrm{abcA}$ & $211,77 \mathrm{bcA}$ \\
Sequeiro & $93,17 \mathrm{aA}$ & $70,54 \mathrm{aB}$ & $90,66 \mathrm{aB}$ & $81,11 \mathrm{aB}$ \\
\hline *em cada coluna, médias seguidas de letras minúsculas diferentes diferem entre si, pelo teste de Tukey, a 5\% de probabilidade; em \\
cada linha, médias seguidas de letras maiúsculas diferentes diferem entre si, pelo teste de Tukey, a 5\% de probabilidade.
\end{tabular}

\section{CONCLUSÕES}

A irrigação e as formas de aplicação de OMM-tech produziram efeitos significativos sobre o rendimento e a produtividade de sementes de pinhão-manso. As plantas irrigadas e adubadas com OMM-Tech pó via solo, na dose de $120 \mathrm{~kg} \mathrm{ha}^{-1}$, foram as que apresentaram a melhor produtividade e rendimento de sementes, respectivamente.

\section{REFERÊNCIAS}

ALLEN, R.G.; PEREIRA, L.S.; RAES, D.; SMITH, M. Crop evapotranspiration: Guidelines for computing crop water requirements. Roma: FAO Irrigation and Drainage, 1998. 297 p. (Paper 56).

AMORIM, D.A.; FAVERO, A.C.; REGINA, M.A. Produção extemporânea da videira, cultivar Syrah, nas condições do sul de Minas Gerais. Revista Brasileira de Fruticultura, Jaboticabal, v.27, n.2, p.327-331, 2005.

ANDRADE, F.V.; MENDONÇA, E.S.; ALVAREZ, V.H.; NOVAIS, R.F. Adição de ácidos orgânicos e húmicos em Latossolos e adsorção de fosfato. Revista Brasileira de Ciência do Solo, Campinas, v.27, p.1.003-1.011, 2003.

ANEZ, L.M.M.; COELHO, M.F.B.; ALBUQUERQUE, M.C.F.; DOMBROSKI, J.L.D. Caracterização morfológica dos frutos, das sementes e do desenvolvimento das plântulas de Jatropha elliptica. Revista Brasileira de Botânica, São Paulo, v.28, n.3, p.563-568, 2005. 
ARIZA-MONTOBBIO, P.; LELE. S. Jatropha plantations for biodiesel in Tamil Nadu, India: Viability, livelihood trade-offs, and latent conflict. Ecological Economics, Amsterdam, v.70, n.2, p.189-195, 2010.

ARRUDA, F.P.; BELTRÃO, N.E.M.; ANDRADE, A.P.; PEREIRA, W.E.; SEVERINO, L.S. Cultivo de pinhão-manso (Jatropha curcas L.) como alternativa para o semiárido nordestino. Revista Brasileira de Oleaginosas e Fibrosas, Campina Grande, v.8, n.1, p.789-799, 2004.

BRASIL. Ministério da Agricultura e Reforma Agrária. Normas Climatológicas (1961-1990). Brasília: Secretaria Nacional de Irrigação, Departamento Nacional de Meteorologia, 1992. 84 p.

CUSTÓDIO, A.A.P.; GOMES, N.M.; LIMA, L.A. Efeito da irrigação sobre a classificação do café. Engenharia Agrícola, Jaboticabal, v.27, n.3, p.691-701, 2007.

DANTAS, A.A.A.; CARVALHO, L.G.; FERREIRA, E. Classificação e tendências climáticas em Lavras-MG. Ciência e Agrotecnologia, Lavras, v.31, p.1.862-1.866, 2007.

DRUMOND, M.A.; SANTOS, C.A.F.; OLIVEIRA, V.R.; MARTINS, J.C.; ANJOS, J.B.; EVANGELISTA, M.R.V. Desempenho agronômico de genótipos de pinhão-manso no Semiárido pernambucano. Ciência Rural, Santa Maria, v.40, n.1, p.44-47, 2010. Disponível em: $<$ http://www.scielo.br/scielo.php?script=sci_abstract\&pid=S0103-84782010000100008 \&lng= en\&nrm=iso\&tlng=pt $>$. Acesso em: 30 mar. 2010.

DRUMOND, M.A.; SANTOS, C.A.F.; OLIVEIRA, V.R.; ANJOS, J.B.; EVANGELISTA, M.R.V. Produção de pinhão-manso no Semiárido brasileiro. In: CONGRESSO INTERNACIONAL DE AGROENERGIA E BIOCOMBUSTÍVEIS, 1., 2007, Teresina. Anais... Teresina: Embrapa Meio Norte, 2007. 1 CD-ROM.

EMBRAPA. EMPRESA BRASILEIRA DE PESQUISA AGROPECUÁRIA. Sistema brasileiro de classificação de solos. Brasília, 1999. 412 p.

FRANCIS, G.; EDINGER, R.; BECKER, K. A concept for simultaneous wasteland reclamation, fuel production, and socio-economic development in degraded areas in India: Need, potencial and perspectives of Jatropha plantations. Natural Resources Forum, New York, v.29, p.12-24, 2005.

LANGE, A.; MARTINES, A.M.; SILVA, M.A.C. da; SORREANO, M.C.M.; CABRAL, C.P.; LAVIOLA, B.G.; DIAS, L.A.S. Teor e acúmulo de nutrientes em folhas e frutos de pinhão-manso. Revista Brasileira de Ciência do Solo, Campinas, v.32, n.5, p.1.969-1.975, 2008.

MALAVOLTA, E. Efeito de deficiência de micronutrientes no estado nutricional da mamoneira cultivar Íris. Pesquisa Agropecuária Brasileira, Brasília, v.40, p.61-67, 2005.

MELO, P.C.; EVANGELISTA, A.W.P.; OLIVEIRA, E.L.; FRAGA, A.C.; CASTRO NETO, P.; FARIA, M.A. Desenvolvimento do pinhão-manso (Jatropha curcas L.) em resposta à aplicação de organominerais-marinho + biotech, fósforo e lâminas de irrigação. In: CONGRESSO

BRASILEIRO DE PLANTAS OLEAGINOSAS, ÓLEOS, GORDURAS E BIODIESEL, 3., 2006, Varginha. Anais... Varginha: UFLA, 2006. 1 CD-ROM.

MELO, P.C.; FURTINI NETO, A.E. Avaliação do lithothamnium como corretivo da acidez do solo e fonte de nutrientes para o feijoeiro. Ciência e Agrotecnologia, Lavras, v.27, n.3, p.508-519, 2003.

MENDONÇA, V.; ORBES, M.Y.; ABREU, N.A.A.; RAMOS, J.D.; TEIXEIRA, G.A.; SOUZA, H.A. Qualidade de mudas de maracujazeiro-amarelo formadas em substratos com diferentes níveis de lithothamnium. Ciência e Agrotecnologia, Lavras, v.30, n.5, p.900-906, 2006.

NÚÑEZ-COLÍN, C.A.; GOYTIA-JIMÉNEZ, M.A. Distribution and agroclimatic characterization of potential cultivation regions of physic nut in Mexico. Pesquisa Agropecuária Brasileira, Brasília, v.44, n.9, p.1.078-1.085, 2009.

OLIVEIRA, E.L.; EVANGELISTA, A.W.P.; MELO, P.C.; FARIA, M.A.; FRAGA, A.C.; CASTRO NETO, P. Crescimento do pinhão-manso em resposta à aplicação de diferentes lâminas 
de irrigação. In: CONGRESSO BRASILEIRO DE PLANTAS OLEAGINOSAS, ÓLEOS, GORDURAS E BIODIESEL, 3., 2008, Varginha. Anais... Varginha: UFLA, 2006. 1 CD-ROM.

PONCIANO DE DEUS, F. Produção de pinhão-manso submetido a diferentes lâminas de irrigação e níveis de adubação potássica. 2010. $81 \mathrm{f}$. Dissertação (Mestrado em Engenharia Agrícola) - Universidade Federal de Lavras, Lavras, 2010.

SATO, F.A.; SILVA, A.M.; COELHO, G.; SILVA, A.C.; CARVALHO, L.G. Coeficiente de cultura $(\mathrm{kc})$ do cafeeiro (Coffea arabica L.) no período de outono-inverno na região de Lavras MG. Engenharia Agrícola, Jaboticabal, v.27, n.2, p.383-391, 2007.

SAVY FILHO, A. Mamona. In: RAIJ, B. van; CANTARELLA, H.; QUAGGIO, J.A.; FURLANI, A.M.C. Recomendações de adubação e calagem para o Estado de São Paulo. Campinas: IAC, 1997. p.201.

SILVA, A.C.; SILVA, A.M.; COELHO, G.; REZENDE, F.C.; SATO, F.A. Produtividade e potencial hídrico foliar do cafeeiro Catuaí, em função da época de irrigação. Revista Brasileira de Engenharia Agrícola e Ambiental, Campina Grande, v.12, n.1, p.21-25, 2008.

SUJATHA, M.; REDDY, T.P.; MAHASI, M.J. Role of biotechnological interventions in the improvement of castor (Ricinus communis L.) and Jatropha curcas L. Biotechnology Advances, New York, v.26, n.5, p.424-435, 2008.

TOMINAGA, N.; KAKIDA, J.; YASUDA, E.K.; SOUZA, L.A.S.; RESENDE, P.L.; SILVA, N.D. Cultivo de pinhão-manso para a produção de biodiesel. Viçosa-MG, CPT, 2007. 220 p. 